\title{
Noise spectrum and signal transmission through a population of spiking neurons
}

\author{
Mona Spiridon and Wulfram Gerstner \\ Center for Neuromimetic Systems, Swiss Federal Institute of Technology, Lausanne, EPFL-DI, \\ CH-1015 Lausanne EPFL, Switzerland
}

Received 28 July 1999

\begin{abstract}
How reliably can a population of spiking neurons transmit a continuous-time signal? We study the noise spectrum of a fully connected population of spiking neurons with relative and absolute refractoriness. Spikes are generated stochastically with a rate that depends on the postsynaptic potential. The analytical solution of the noise spectrum of the population activity is compared with simulations. We find that strong inhibitory couplings can considerably reduce the noise level in a certain frequency band. This allows the population to reliably transmit signals at frequencies close to or even above the single-neuron firing rate.
\end{abstract}

\section{Introduction}

Reaction time experiments [27] have shown that humans can visually recognize objects in a few hundreds of milliseconds. Considering that the signal has to pass through different processing stages, the neurons at each processing stage must transmit the signal in tens of milliseconds. Cortical neurons fire with a rate that does not exceed a few hundred spikes per second. This means that, at each processing stage, a neuron has the time to emit one or two spikes only. A code based on the temporal average of input spikes cannot correctly describe fast information transmission.

The presence of noise renders information transmission even more complicated. Spike patterns of cortical neurons are highly variable and in most cases are not reproducible from trial to trial. In many cortical areas, the interspike interval is close to a Poisson distribution [26]. The origin of noise in neuronal spiking is not yet clear. Experiments have shown that neurons have a low intrinsic noise level $[7,20]$. Most probably, noise is due to the stochastic arrival times of the input spikes which may arise in a network with balanced excitation and inhibition [6,32]. Thus, the irregular spiking is an emergent property of the network rather than an intrinsic property of the single neuron. It has been shown, however, that noise in the spike arrival time can be approximated by intrinsic neuronal noise [14,23]. The advantage of intrinsic noise is that it facilitates the analysis of the population dynamics. Theoretical studies $[2,13]$ have also shown that the presence of noise may be useful for a network to stabilize its asynchronous activity.

Thus, the brain seems to use a coding principle that (i) allows fast transmission and (ii) is noise resistant. The spatially averaged population activity (which corresponds to a population rate) seems to be such a natural coding principle. A population activity based code has two advantages. First, the population activity can react very fast to changes in the 
input $[14,18,30,32]$. Second, noise in the system can be averaged out among redundant neurons.

In many areas of the brain, neurons are organized in pools of neurons with similar properties (i.e columns in the visual cortex or pools of motor neurons). Averaging over $N$ uncoupled neurons improves the signal-to-noise ratio (SNR) by a factor of $1 / \sqrt{N}$. Biological neurons, however, are connected to each other. How does spatial averaging change if neurons are not independent but interconnected? Here, we study the influence of network parameters (coupling strength, delay, refractory period, etc) on the signal transmission reliability. We present a theory that describes the noise properties of a network of stochastic spiking neurons in a fully connected network. An analytical expression of the noise power and the SNR is given for each frequency and as a function of the network parameters.

We find that anticorrelations in the firing shift the noise from low frequency to high frequency. This phenomenon, called spectral noise shaping, is well known in the technology of $\mathrm{A} / \mathrm{D}$ converters and has been proposed by Adams as a potential neuronal coding principle $[4,21]$. Noise power is moved from the frequency band used for signal transmission to a different frequency range through appropriate feedback. We show here that inhibitory connections can produce significant noise shaping over a bandwidth that, for a low level of activity $(1-10 \mathrm{~Hz})$, extends to frequencies above the mean firing rate of a single neuron.

\section{Theory}

\subsection{The spike response model}

The state of a neuron $i$ is described by a variable $u_{i}$ which represents the membrane potential of neuron $i$. If $u_{i}$ reaches the threshold $\vartheta$, a spike is emitted. The moment of threshold crossing defines the firing time $t_{i}^{(f)}$. After the spike, the neuron undergoes a refractory period that is mathematically represented by a negative contribution $\eta_{i}\left(t-t_{i}^{(f)}\right)$ to the membrane potential $u_{i}$. Each presynaptic spike induces, after an axonal delay $\Delta_{\mathrm{ax}}$, a change in the membrane depolarization. The effect of spike arrival is an excitatory or inhibitory postsynaptic potential (EPSP or IPSP). The time course of such a postsynaptic potential (PSP) is represented by the kernel $\varepsilon\left(t-t_{j}^{(f)}-\Delta_{\mathrm{ax}}\right)$ where $t_{j}^{(f)}$ is the presynaptic firing time. The total PSP, $h_{i}(t)$, is a linear superposition of the PSPs summed over all neurons and over all firing times, weighted by a coupling factor $w_{i j}$

$$
h_{i}(t)=\sum_{j=1}^{N} \sum_{t_{j}^{(f)}} w_{i j} \varepsilon\left(t-t_{j}^{(f)}-\Delta_{\mathrm{ax}}\right)+h_{\mathrm{ext}}(t) .
$$

The second term of the rhs of equation (1) is the PSP produced by some external input $\mathcal{I}_{\text {ext }}(t)$, filtered by the kernel $\varepsilon_{\text {ext }}$ :

$$
h_{\text {ext }}(t)=J_{\text {ext }} \int_{0}^{\infty} \varepsilon_{\text {ext }}(s) \mathcal{I}_{\text {ext }}(t-s) \mathrm{d} s .
$$

The membrane potential can be written as

$$
u_{i}\left(t \mid \hat{t}_{i}\right)=\eta_{i}\left(t-\hat{t}_{i}\right)+h_{i}(t)
$$

where $\hat{t}_{i}$ is the last firing of neuron $i$, i.e. $\hat{t}_{i}=\max \left\{t_{i}^{(f)} \mid t_{i}^{(f)}<t\right\}$. The function $\eta_{i}\left(t-\hat{t}_{i}\right)$ describes the effect of the last firing $\hat{t}_{i}$ on the membrane potential.

We consider a simplified neuronal network. All neurons are similar so we can drop the indices $i$ on the kernel $\eta$ and the synaptic strength is identical for all neurons:

$$
w_{i j}=\frac{J}{N} \text {. }
$$


The interaction strength scales with the reciprocal of $N$, the number of neurons, so that the total input remains finite for $N \rightarrow \infty$.

The kernels $\varepsilon\left(t-t_{j}^{(f)}-\Delta_{\mathrm{ax}}\right), \varepsilon_{\mathrm{ext}}(s)$ and $\eta\left(t-\hat{t}_{i}\right)$ can be chosen arbitrarily. For the simulations, we take:

$$
\begin{aligned}
& \eta(s)=-\eta_{0} \exp \left(-\frac{s-\delta_{\mathrm{abs}}}{\tau_{m}}\right) \mathcal{H}\left(s-\delta_{\mathrm{abs}}\right)+K \mathcal{H}\left(\delta_{\mathrm{abs}}-s\right) \\
& \varepsilon_{\mathrm{ext}}(s)=\frac{1}{\tau_{m}} \exp \left(-\frac{s}{\tau_{m}}\right) \mathcal{H}(s) \\
& \varepsilon(s)=\frac{1}{\tau_{s}-\tau_{m}}\left[\exp \left(-\frac{s}{\tau_{s}}\right)-\exp \left(-\frac{s}{\tau_{m}}\right)\right] \mathcal{H}(s)
\end{aligned}
$$

where $\tau_{s}$ and $\tau_{m}$ are, respectively, the synaptic and the membrane time constant. $\delta_{\text {abs }}$ is the absolute refractory period and $K$ is a large negative constant. We set $\eta_{0}=1$. To ensure causality, we have added the Heaviside function $\mathcal{H}(\cdot)$ with $\mathcal{H}(s)=1$ for $s>0$ and 0 elsewhere. The spike response model with kernels (5)-(7) is, at least for low firing rates, equivalent to the integrate-and-fire model $[13,15]$.

For $\tau_{m}=\tau_{s}=\tau$, equation ( 7 ) reduces to

$$
\varepsilon(s)=\frac{s}{\tau^{2}} \exp \left(-\frac{s}{\tau}\right) \mathcal{H}(s)
$$

\subsection{Noise model}

We assume that a neuron can fire even though the formal threshold has not been reached. To do so we introduce an escape rate $\rho$ that depends on the difference between the present value of the membrane potential $u\left(t \mid \hat{t}_{i}\right)$ and the threshold $\vartheta$ :

$$
\rho_{h_{i}}\left(t \mid \hat{t}_{i}\right)=f\left[u\left(t \mid \hat{t}_{i}\right)-\vartheta\right]=f\left[\eta\left(t-\hat{t}_{i}\right)+h_{i}(t)-\vartheta\right]
$$

The second equality follows from (3). $f$ is an arbitrary function. Here we use either a piecewise linear function $\rho=\rho_{0} \cdot(u-\vartheta) \mathcal{H}(u-\vartheta)$ or a Gaussian distribution $\rho=\alpha \cdot \exp \left[(u-\vartheta)^{2} / 2 \beta^{2}\right]$ for $u \leqslant \vartheta$ and $\rho=\alpha$ for $u>\vartheta$, where $\rho_{0}, \alpha$ and $\beta$ are parameters. In most of our simulations, the potential $u$ rests slightly below threshold so that the definition of the Gaussian escape rate $\rho$ for $u>\vartheta$ (which is somewhat arbitrary) is not important. For a motivation of escape rate models see [23] where different choices of escape function are discussed.

One of the advantages of this noise model (equation (9)) is that we can calculate explicitly the distribution of interspike intervals. Let us suppose that neuron $i$ has emitted its last spike at $\hat{t}_{i}$. If we know the external input $h_{\text {ext }}$ and the firing times $t_{j}^{(f)}<t$ of presynaptic neurons $j$, we can calculate the input potential $h_{i}(t)$ from equation (1) and hence the membrane potential $u_{i}\left(t \mid \hat{t}_{i}\right)$ from equation (3). What is the probability that the neuron, after having emitted a spike at $\hat{t}_{i}$, does not emit a spike up to time $t$ ? The probability is given by the 'survivor' function $S_{h_{i}}\left(t \mid \hat{t}_{i}\right)=\exp \left\{-\int_{\hat{t}_{i}}^{t} \rho_{h_{i}}\left(s \mid \hat{t}_{i}\right) \mathrm{d} s\right\}$, where $\rho_{h_{i}}\left(t \mid \hat{t}_{i}\right)=f\left[\eta\left(t-\hat{t}_{i}\right)+h_{i}(t)-\vartheta\right][16]$. The survivor function obviously depends on $\hat{t}_{i}$, the last firing time of neuron $i$. The lower index $h_{i}$ in the survivor function is intended to remind the reader that $S_{h_{i}}\left(t \mid \hat{t}_{i}\right)$ also depends on the input potential $h_{i}(t)$. The probability density $P_{h_{i}}\left(t \mid \hat{t}_{i}\right)$ that the next spike of neuron $i$ occurs at $t$ is $\rho_{h_{i}}\left(t \mid \hat{t}_{i}\right) \cdot S_{h_{i}}\left(t \mid \hat{t}_{i}\right)$. Hence

$$
P_{h_{i}}\left(t \mid \hat{t}_{i}\right)=\rho_{h_{i}}\left(t \mid \hat{t}_{i}\right) \exp \left[-\int_{\hat{t}_{i}}^{t} \rho_{h_{i}}\left(s \mid \hat{t}_{i}\right) \mathrm{d} s\right]
$$


Given the last spike at $\hat{t}_{i}$ and an input potential $h_{i}(t)$ for $t>\hat{t}_{i}$, the probability that the next spike occurs between $t$ and $t+\Delta t$ is $P_{h_{i}}\left(t \mid \hat{t}_{i}\right) \cdot \Delta t$. We note that

$$
P_{h_{i}}\left(t \mid \hat{t}_{i}\right)= \begin{cases}-\frac{\partial}{\partial t} S_{h_{i}}\left(t \mid \hat{t}_{i}\right) & \text { for } t>\hat{t}_{i} \\ 0 & \text { otherwise. }\end{cases}
$$

\subsection{Population activity}

The population activity is defined as

$$
A(t)=\lim _{\Delta t \rightarrow 0} \frac{1}{\Delta t} \frac{n_{\text {act }}(t ; t+\Delta t)}{N}=\frac{1}{N} \sum_{t^{(f)}} \delta\left(t-t^{(f)}\right)
$$

where $N$ is the size of the population, $n_{\text {act }}(t ; t+\Delta t)$ is the number of neurons that fire during the interval $\Delta t$, and $t^{(f)}$ is the firing time of a pulse. The sum runs over all firings of all neurons in the population. The activity (12) corresponds to a spatially averaged population rate. In contrast to a temporally averaged mean firing rate, the population rate can, in principle, respond quickly to changes in the input $[14,18,30,32]$. Using the activity (12) in the form $A(t)=N^{-1} \sum_{t^{(f)}} \delta\left(t-t^{(f)}\right)$, we can rewrite equation (1) as

$$
h(t)=J \int_{0}^{\infty} \varepsilon(s) A(t-s) \mathrm{d} s+h_{\text {ext }}(t) .
$$

Note that, compared with equation (1), the lower index $i$ has been suppressed since all neurons receive the same total PSP.

\section{Theoretical results}

\subsection{Noise spectrum}

The population activity dynamics in a homogeneous network of spiking neurons can be written as $[13,14]$ :

$$
A(t)=\int_{-\infty}^{t} P_{h}(t \mid \hat{t}) A(\hat{t}) \mathrm{d} \hat{t}
$$

where the kernel $P_{h}(t \mid \hat{t})$ is the probability density that a neuron that has spiked at time $\hat{t}$ and that is subject to a potential $h(t)$ will produce another spike at time $t$; see equation (10). The present activity $A(t)$ depends on the past activity $A(\hat{t})$ for $\hat{t}<t$, since the number of neurons that have fired around $\hat{t}$ is proportional to $A(\hat{t})$. For the sake of clarity, we rewrite the probability density $P_{h}(t \mid \hat{t})$, expressed as a function of the escape rate $\rho_{h}(t \mid \hat{t})$ :

$$
P_{h}(t \mid \hat{t})=\rho_{h}(t \mid \hat{t}) \exp \left[-\int_{\hat{t}}^{t} \rho_{h}(s \mid \hat{t}) \mathrm{d} s\right]
$$

where $\rho_{h}(t \mid \hat{t})=f[\eta(t-\hat{t})+h(t)-\vartheta]$. The only difference to (10) is that we have omitted, in (15), the index $i$ since $h(t)$ is independent of $i$; $\operatorname{cf}(13)$.

We calculate the activity and the noise spectrum for a constant external input $h_{\mathrm{ext}}(t)=h_{\mathrm{e}}$. For $N \rightarrow \infty$ and sufficiently high noise, the activity $A(t)$ tends to a fixed point $A_{0}$. The value of $A_{0}$ has to be determined self-consistently from $A_{0}=\left(\int_{0}^{\infty} \mathrm{d} s \exp \left[-\int_{0}^{s} \mathrm{~d} s^{\prime} \rho_{h_{0}}\left(s^{\prime} \mid 0\right)\right]\right)^{-1}$ where $h_{0}=J A_{0}+h_{\mathrm{e}}$ is the constant term of the PSP (see [13,14]). Due to the finite number of neurons, $A(t)$ will fluctuate around $A_{0}$. Each of the $N$ neurons fires stochastically with its 
momentary rate $\rho_{h}(t \mid \hat{t})$. We replace the $N$ stochastic processes by a single one and introduce a coherent fluctuation of the $N$ escape rates:

$$
\rho_{h}(t \mid \hat{t}) \rightarrow \rho_{h}(t \mid \hat{t})[1+\sigma \xi(t)]
$$

where $\xi$ is a Gaussian white noise with zero mean and autocorrelation $\left\langle\xi(t) \xi\left(t^{\prime}\right)\right\rangle=\delta\left(t-t^{\prime}\right)$. Equation (16) is somewhat 'ad hoc' and would be difficult to justify rigorously. Qualitatively, the idea is that, in each interval $\Delta t$, the expected number of active neurons is $N \cdot A_{0} \cdot \Delta t$. Due to finite size, fluctuations will be of order $\sqrt{N A_{0} \Delta t}$. Hence, the relative fluctuations can be approximated by setting $\sigma=1 / \sqrt{N A_{0}}$ in equation (16). For large $N$, the parameter $\sigma$ is small.

We linearize the activity around its fixed point $A(t)=A_{0}+\Delta A(t)$. Expansion of equation (14) to first order in $\Delta A$ and $\sigma$ gives the following equation:

$$
\begin{gathered}
\Delta A(t)=\int_{-\infty}^{t} \mathrm{~d} \hat{t} P_{h_{0}}(t \mid \hat{t}) \Delta A(\hat{t})+A_{0} \frac{\mathrm{d}}{\mathrm{d} t}\left[\int_{0}^{\infty} \mathrm{d} x \mathcal{L}(x)\left(h(t-x)-h_{0}\right)\right] \\
+\sigma A_{0} \frac{\mathrm{d}}{\mathrm{d} t}\left[\int_{0}^{\infty} \mathrm{d} x \mathcal{F}(x) \xi(t-x)\right] .
\end{gathered}
$$

The first term represents the influence of past perturbations. The second term describes the variations in the activity due to the fluctuations in the PSP, $h(t)$. The last term is the noise due to the finite population size. A more detailed derivation of equation (17) is presented in the appendix. The functions $\mathcal{F}(t)$ and $\mathcal{L}(t)$ are defined as

$$
\begin{aligned}
& \mathcal{F}(t)=\mathcal{H}(t) \int_{t}^{\infty} \rho_{h_{0}}(s-t) S_{h_{0}}(s) \mathrm{d} s \\
& \mathcal{L}(t)=\mathcal{H}(t) \int_{t}^{\infty}\left[\frac{\mathrm{d}}{\mathrm{d} h_{0}} \rho_{h_{0}}(s-t)\right] S_{h_{0}}(s) \mathrm{d} s .
\end{aligned}
$$

For $h(t) \equiv h_{0}$, the escape rate $\rho(t \mid \hat{t})$ and hence the survivor function $S(t \mid \hat{t})$ depend only on the time difference $t-\hat{t}$. We set $\rho_{h_{0}}(t-\hat{t})=\rho_{h_{0}}(t \mid \hat{t})$ and $S_{h_{0}}(t-\hat{t})=S_{h_{0}}(t \mid \hat{t})$.

Taking the Fourier transform of equation (17) and using (11) gives an expression of noise spectrum:

$$
\left|\tilde{A}_{\text {noise }}(\omega)\right|^{2}=\frac{\sigma^{2} A_{0}^{2}|\tilde{\mathcal{F}}(\omega)|}{\left|\tilde{S}_{h_{0}}(\omega)-J A_{0} \tilde{\mathcal{L}}(\omega) \tilde{\varepsilon}(\omega)\right|^{2}}|\tilde{\xi}(\omega)|^{2}
$$

where $\sigma^{2}=\left(1 / A_{0} N\right)$. For finite $\Delta t$, we use $|\tilde{\xi}(\omega)|^{2}=1 / \Delta t$. The tilde denotes the Fourier transform.

\subsection{Absolute refractoriness only}

For absolute refractoriness of length $\delta_{\mathrm{abs}}$ and without any relative refractory period, namely $\eta_{0}=0$ in equation (5), the noise spectrum reduces to

$$
\left|\tilde{A}_{\text {noise }}(\omega)\right|^{2}=\frac{\left(A_{0} / N\right)}{\left|1-\left[1-A_{0} \delta_{\text {abs }}\right] \rho^{\prime} J \tilde{\varepsilon}(\omega)+\rho_{h_{0}} \tilde{\mathbf{1}}_{\left[0, \delta_{\text {abs }}\right]}(\omega)\right|^{2}}|\tilde{\xi}(\omega)|^{2}
$$

where the tilde denotes the Fourier transform and $\mathbf{1}_{\left[0, \delta_{\text {abs }}\right]}$ denotes the indicator function which is unity on the interval $\left[0, \delta_{\mathrm{abs}}\right]$ and vanishes otherwise. $\rho^{\prime}$ is the derivative of the escape rate with respect to the membrane potential evaluated at $h_{0}$. For finite $N$ and in the absence of coupling $(J=0)$, the spectrum shows the effect of refractoriness. In this case, it reduces to the spectrum calculated in [12]. In the limit of no refractoriness $\left(\delta_{\mathrm{abs}}=0\right)$, equation (21) reduces to the Hawkes formula for coupled point processes [10]. For finite $\Delta t,|\tilde{\xi}(\omega)|^{2}=1 / \Delta t$. 


\subsection{Signal spectrum}

Let us consider the case when the neuronal population receives an external current $I_{\mathrm{ext}}(t)$ that varies over time. The output signal spectrum can be derived from equation (17) by taking the Fourier transform of the first two terms (see [14]):

$$
\left|\tilde{A}_{\text {signal }}(\omega)\right|^{2}=\frac{A_{0}{ }^{2}|\tilde{\mathcal{L}}(\omega)|^{2}}{\left|\tilde{S}_{h_{0}}(\omega)-J A_{0} \tilde{\mathcal{L}}(\omega) \tilde{\varepsilon}(\omega)\right|^{2}} J_{\text {ext }}^{2}\left|\tilde{\varepsilon}_{\text {ext }}(\omega) \tilde{I}_{\text {ext }}(\omega)\right|^{2} .
$$

The SNR is thus given by the ratio between the output signal (equation (22)) and the noise term (equation (20)):

$$
|\operatorname{SNR}(\omega)|^{2}=\frac{|\tilde{\mathcal{L}}(\omega)|^{2}}{\sigma^{2}|\tilde{\mathcal{F}}(\omega)|^{2}|\tilde{\xi}(\omega)|^{2}} J_{\text {ext }}^{2}\left|\tilde{\varepsilon}_{\text {ext }}(\omega) \tilde{I}_{\text {ext }}(\omega)\right|^{2} .
$$

\section{Simulation results}

\subsection{The theory compared with simulations}

In order to validate our theory, we simulate a network of 1000 neurons described by the model presented in section 2.1. Each neuron receives the same constant external input $h_{\mathrm{e}}$ and positive or negative feedback from the other neurons in the network, with a synaptic efficiency $J / N$. Neurons have an absolute and a relative refractory period described by the kernel $\eta$ (equation (5)). The activity is measured for $10 \mathrm{~s}$ with a time step of $\Delta t=0.1 \mathrm{~ms}$. At each time step, neurons fire with a spiking probability $\Delta t \cdot f[u(t \mid \hat{t})-\vartheta]$, where $f$ is either a piecewise linear function or the Gaussian function introduced in section 2.2. Note that there is no free parameter, since $\sigma^{2}=\left(1 / A_{0} N\right) ; A_{0}=\int s P_{h_{0}}(s \mid 0) \mathrm{d} s$ and $P_{h_{0}}(t \mid \hat{t})$ is given by equation (15).

Figure 1 compares the theoretical spectrum for a linear escape rate with the one obtained from a simulation in the absence of connections $(a)$, and in the case of a fully connected network with inhibitory connections $(b)$. In both cases the theory is in excellent agreement with the simulations. The theory, which has been elaborated for a fully connected network, also describes very well the noise spectrum for a partially connected network. In this case, the coupling strength is adjusted so that the total synaptic weight received by each neuron, $\sum_{j} J_{i j}=J$, is identical for each neuron $i$ (figure 1(c)). The good agreement between the theory and the simulations remains valid if the linear spiking probability is replaced by a Gaussian distribution (figures $1(d)-(f)$ ).

\subsection{Effects of the network parameters on the noise spectrum}

We have investigated how the different network parameters influence the noise characteristics of a population of spiking neurons. The effect of the axonal delay and the PSP time constants on the noise spectrum are briefly described in the discussion. For the sake of simplicity, below we present the theoretical noise spectrum using a linear escape rate. For Gaussian escape rates, the effects would be similar.

4.2.1. Refractory period. In the absence of coupling and of refractoriness, the noise spectrum is completely flat as one would expect for a Poisson process. Adding a refractory period causes a dip in the power spectra in the low-frequency range. Recorded spike trains from cortical neurons also present this manifestation of refractoriness in the frequency content $[5,17]$. Increasing the refractory period reduces the noise amplitude at low frequency and shifts the first peak of the spectrum towards lower frequency (figure 2(a)). The location of the peaks 
a)

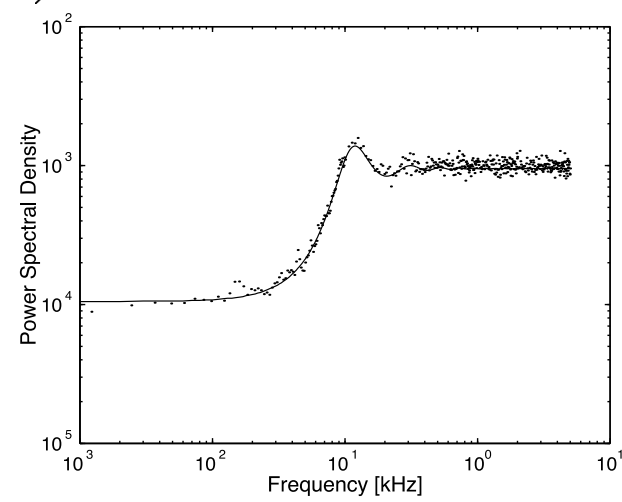

b)

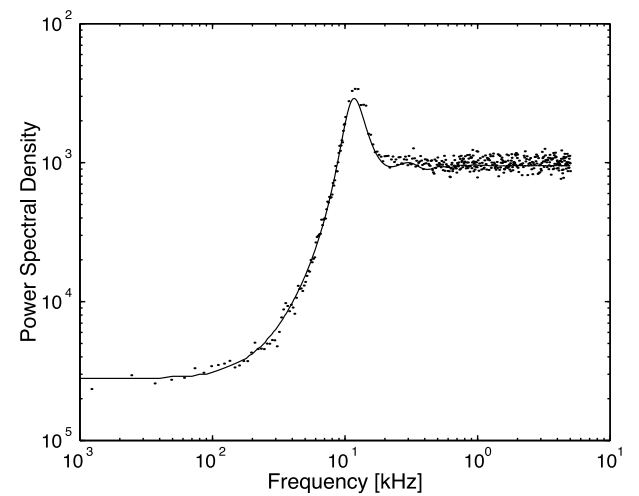

c)

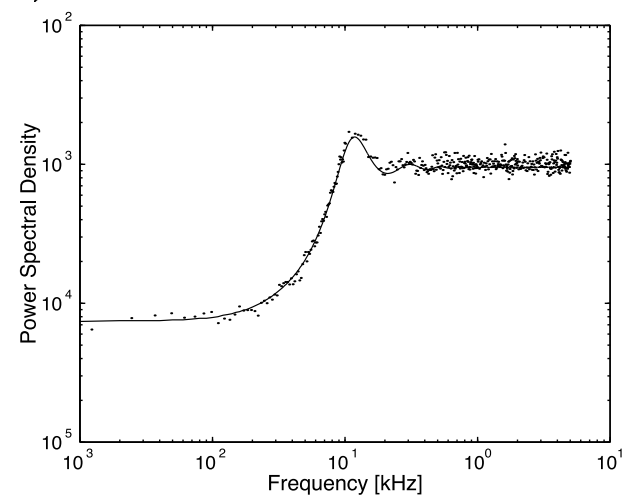

d)

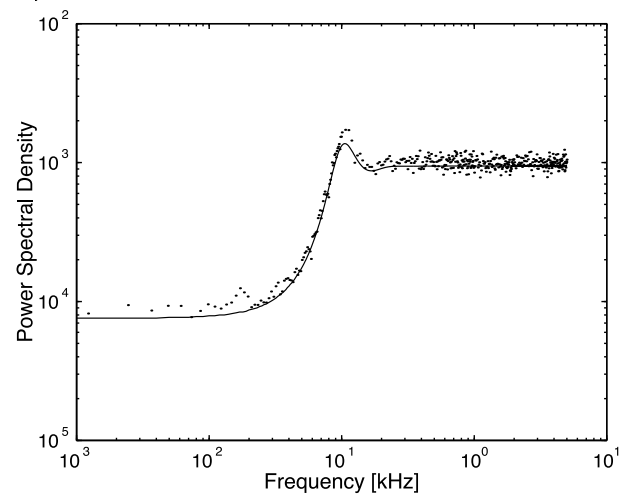

e)

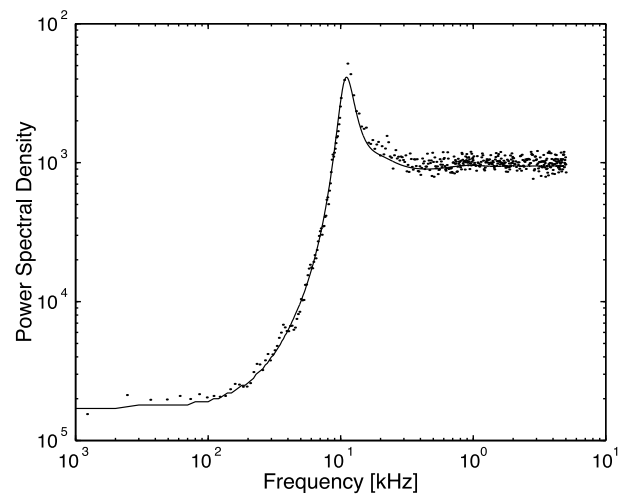

f)

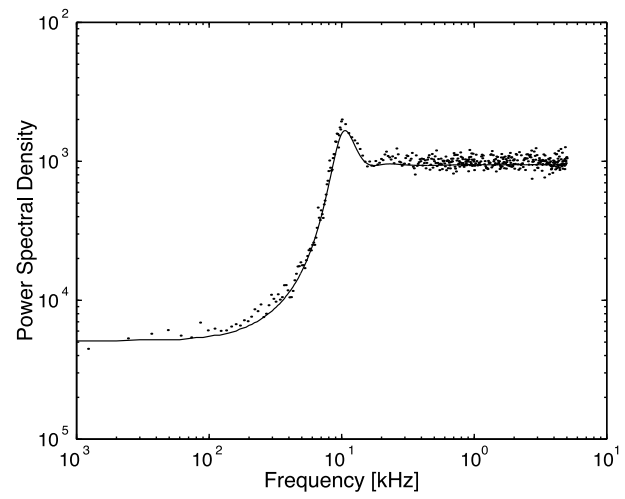

Figure 1. Theoretical noise spectrum (solid curves) and noise spectrum obtained from simulations of a network with 1000 neurons (points). $(a)-(c)$ Linear escape rate with slope $\rho_{0}=1$. $(d)-(f)$ Gaussian escape rate with variance $\beta^{2}=0.05$ and parameter $\alpha=1$. (a), (d) Network without coupling $(J=0)$. (b), (e) Inhibitory coupling $(J=-5) .(c),(f)$ simulations (points) with a partially connected network and inhibitory coupling strength $w_{i j}=-1 / 200$. Each neuron is connected to 200 out of 1000 neurons in the network. The theoretical spectrum (solid curve) of a fully connected network that reproduces the spectrum of the partially connected network has a coupling strength of $J=-1$. The following network parameters are the same for all the figures: the axonal delay and the absolute refractory period are equal to $1 \mathrm{~ms}$, the mean activity is $100 \mathrm{~Hz}$ and the membrane and synaptic time constants are equal to $4 \mathrm{~ms}$. 
a)

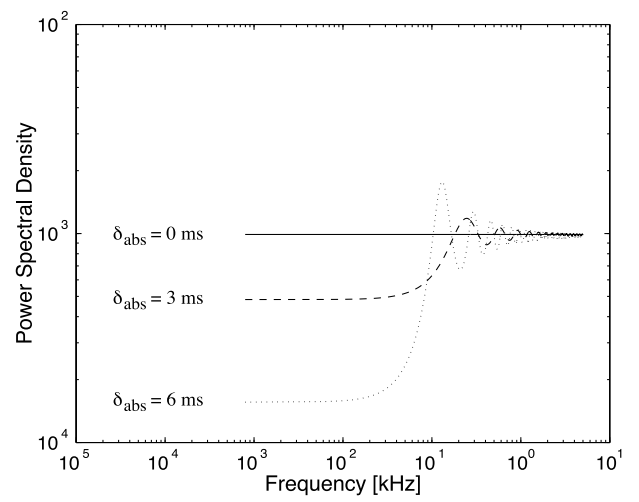

b)

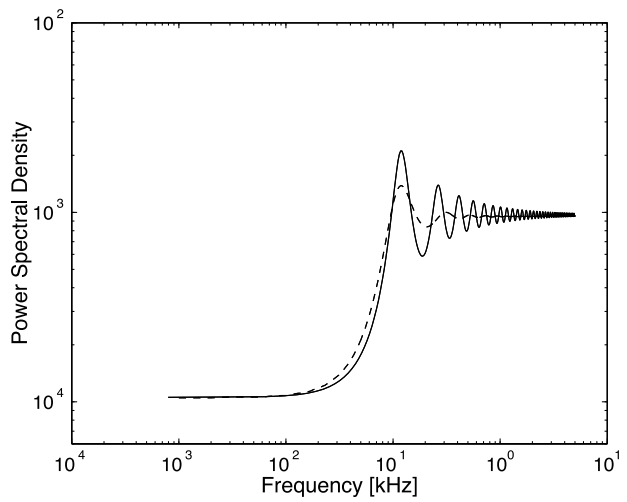

Figure 2. (a) Theoretical noise spectrum for different values of the absolute refractory periods $\delta_{\text {abs }}$ and in the absence of coupling $(J=0)$. The transmission delay is equal to $1 \mathrm{~ms}$. The escape rate $\rho(t)$ depends linearly on the input. The synaptic and membrane time constants are equal to $4 \mathrm{~ms}$. (b) Theoretical noise spectrum in the absence of coupling for two types of refractory periods. The solid curve corresponds to an absolute refractory period $\delta_{\mathrm{abs}}=6.7 \mathrm{~ms}$ and no relative refractory period and the dashed curve to a relative refractory period with $\tau_{m}=4 \mathrm{~ms}$ and no absolute refractory period. The other parameter values are the same as in $(a)$. In all cases, the background input $h_{e}$ was adjusted so that the mean activity is always $A_{0}=100 \mathrm{~Hz}$.

varies inversely with the refractory period. In figure $2(b)$ we compare the noise spectra in two cases: neurons having only an absolute refractory period, and neurons having a relative refractory period with a time constant of $4 \mathrm{~ms}$. We use the absolute refractory period as a free parameter to optimize the noise spectrum in the low-frequency band (up to $100 \mathrm{~Hz}$ ). The optimization gives an absolute refractory period of $6.7 \mathrm{~ms}$. Figure $2(b)$ shows that replacing the absolute refractory period by relative refractoriness reduces the peak amplitudes. Thus, for the same mean activity, the relative refractoriness contributes to the stability the network. The spectra of uncoupled neurons can also be calculated directly from renewal theory [9].

4.2.2. Coupling strength. In order to compare results performed with different coupling strengths $J$, we adjust the external input $h_{\mathrm{e}}$ so as to fix the mean activity at a value of $100 \mathrm{~Hz}$. This value corresponds to a mean interspike interval of $10 \mathrm{~ms}$. We see from figure 3(a) that for $J$ large and positive, the asynchronous state would become instable (large peaks in the power density). Inhibitory connections $(J<0)$ shifts the noise from the low to the high-frequency band (figure 3(a)). For large inhibitory connections $(J=-10)$, the noise level for frequencies $<10 \mathrm{~Hz}$ can be reduced by a factor of 100 , compared with the one at high frequency.

4.2.3. Mean activity. Different neuronal populations in the brain have different mean spontaneous activities. Here, we study the effect of the mean activity on the noise power. For a homogeneous population, the mean activity corresponds to the mean firing rate of a single neuron. We compare this firing rate with the bandwidth of the low-noise regime. In figure $3(b)$, we plot the noise spectrum for different mean activities. The arrows represent the mean firing rate of a single neuron. For high levels of activity $(\sim 100 \mathrm{~Hz})$, the bandwidth of noise reduction does not extend to the firing rate of a single neuron. On the other hand, at low activity level $(1-10 \mathrm{~Hz})$, the noise reduction extends beyond the single-neuron frequency. As a consequence, anticorrelations in the firings can allow signals, faster than the single neuron frequency, to be transmitted with high SNR. 
a)

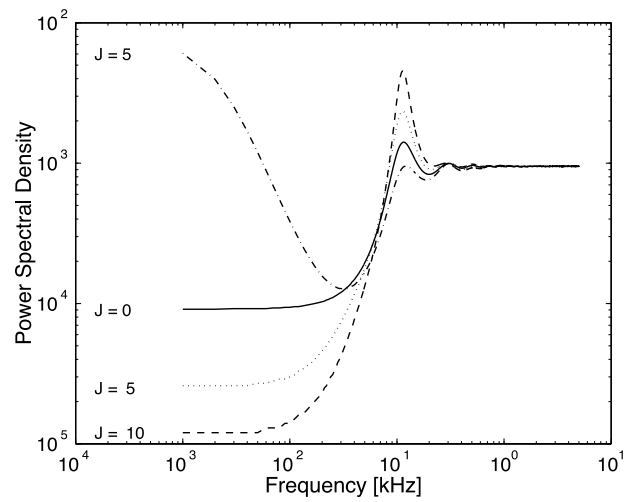

b)

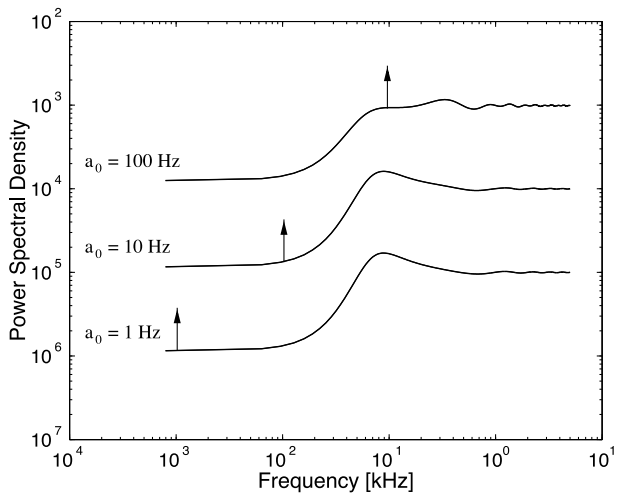

Figure 3. (a) Theoretical noise spectra for different coupling strengths $J$. The membrane time constant $\tau_{m}$ and the synaptic time constant $\tau_{s}$ are equal to $5 \mathrm{~ms}$. The mean activity $A_{0}$ is $100 \mathrm{~Hz}$ and the transmission delay is equal to $1 \mathrm{~ms}$. The escape rate $\rho(t)$ depends linearly on the input. The refractory period is composed of an absolute refractory period of $1 \mathrm{~ms}$ and a relative refractory period. (b) Theoretical noise spectra for different mean activities $A_{0}$. The arrows represent the mean firing rate of a single neuron. The time constants are $\tau_{m}=4 \mathrm{~ms}$ and $\tau_{s}=4 \mathrm{~ms}$. The transmission delay is equal to $1 \mathrm{~ms}$. The escape rate $\rho(t)$ depends linearly on the input. The absolute refractory period is equal to $2 \mathrm{~ms}$ and there is no relative refractoriness. The coupling factor $J=-2$.

\subsection{Noisy input model}

The model that we have used is an oversimplification of biological neurons. To show that the main characteristics of the spectrum are not dependent on this model, we have performed additional simulations with a different noise model that is more closely related to biology. In cortical neurons, noise probably results primarily from the variability in the spike arrival times. The membrane potential is close to the threshold most of the time and spikes are triggered by random fluctuations in the input $[6,29,32]$. Using the spike response model to describe the neuronal state, the membrane potential of a neuron $i$, subject to stochastic spike arrival, can be written as

$u_{i}(t)=\eta_{i}\left(t-t_{i}^{(f)}\right)+\frac{J}{N} \sum_{j} \sum_{t_{j}^{(f)}} \varepsilon\left(t-t_{j}^{(f)}-\Delta_{\mathrm{ax}}\right)+\frac{J_{\mathrm{ext}}}{N} \sum_{t^{\prime} \in M_{i}} \varepsilon_{\mathrm{ext}}\left(t-t^{\prime}-\Delta_{\mathrm{ax}}\right)$

where the set $M_{i}$ of firing times $t^{\prime}$ is generated by a Poisson process and is different for each neuron. The last term corresponds to some stochastic external input. When the membrane potential reaches the threshold $\vartheta$, a spike is emitted.

We consider the noise spectrum of the noisy input model defined by equation (24). Figure 4(a) shows the noise spectrum resulting from the simulations of such a network without connections $(J=0)$ and with inhibitory connections $(J=-5)$. Inhibitory coupling with strength $J=-5$ reduces the noise, approximatively, by a factor of two in the low-frequency band. The theory elaborated for stochastic neurons with noisy threshold (escape rate model) is compared with the noise spectrum of the noisy input model (figure $4(b)$ ). For the theory, we use a linear escape rate and adjust the parameter $\rho_{0}$ to obtain a good fit at low frequency. At high frequency, the noise level depends on the mean activity which is the same for the theory and simulations. The results show a significant shift between the theoretical spectrum and the simulation with the noisy input model (figure $4(b)$ ). However, the main features of the noisy input spectrum are reproduced by the theory. 
a)

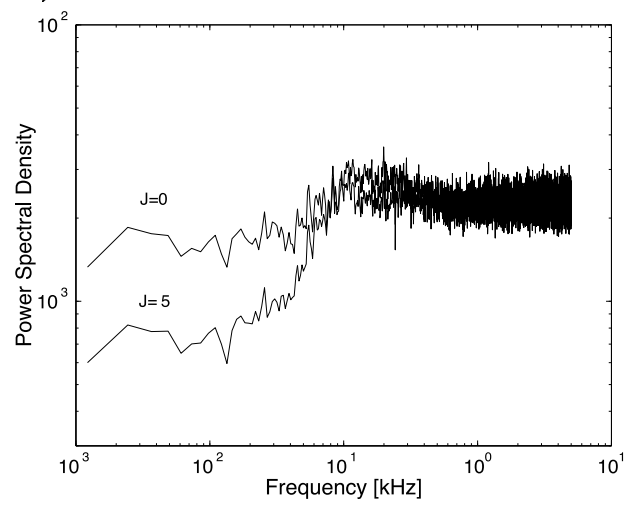

b)

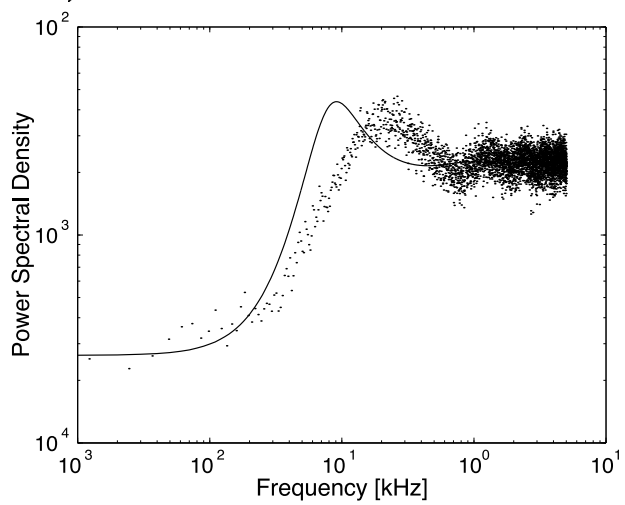

Figure 4. (a) Noise spectra for two coupling strengths with the noisy input model. Inhibitory couplings $(J=-5)$ reduce the noise at low frequency. The network has 100 neurons with an absolute refractory period and an axonal delay of $1 \mathrm{~ms}$. Each neuron receives a different Poissonian spike train with a mean rate of $1 \mathrm{kHz}$. The external input strength is adjusted in order to produce a mean activity of $23 \mathrm{~Hz}$ in both cases $\left(J_{\text {ext }}=80\right.$ for the network with no couplings and $J_{\text {ext }}=89$ for the network with inhibitory couplings). (b) Noise spectrum from the simulations of the noisy input model (points) and theoretical noise spectrum with the escape rate model (solid curve). The internal coupling strength is equal to $J=-5$ and the axonal delay is equal to $1 \mathrm{~ms}$. The membrane and synaptic time constant are, respectively, $\tau_{m}=5 \mathrm{~ms}$ and $\tau_{s}=2 \mathrm{~ms}$. Each neuron receives a different Poissonian external spike train with a $10 \mathrm{kHz}$ mean rate and synaptic efficacy $J_{\text {ext }}=7.3$. In both cases (theory and simulations) the mean activity is equal to $22.5 \mathrm{~Hz}$ and the parameter $\rho_{0}$ of the linear escape rate has been adjusted to best fit the simulations.

\section{Signal transmission}

\subsection{SNR}

The reliability of signal transmission may be characterized by the SNR. Equation (23) gives the ratio between the output signal and the output noise for a given frequency. The ratio does not depend explicitly on the internal coupling strength. In order to compare the SNR for different coupling strengths, it is reasonable to keep the output signal amplitude constant. To achieve this, the external coupling strength $\left(J_{\text {ext }}\right)$ has been adjusted. Indeed, let us suppose that the neuronal population receives an external input that oscillates with a certain frequency. To transmit the signal in the most reliable manner, the input signal should be amplified as much as possible (by means of $J_{\text {ext }}$ ). However, there is a limit in the amplification of the signal in order to maintain the system in a stable and, possibly, linear regime. This limit depends on the internal structure (or parameters) of the network.

Figure 5 illustrates the above argument. A sinusoidal input (figure 5(a)) is applied to a network without connections (figure $5(b)$ ) and a fully connected inhibitory network $(J=-5)$ (figure $5(c)$ ). For the same amplification (same $J_{\text {ext }}$ ), the system is, in the absence of coupling, clearly in a nonlinear regime, whereas, for $J=-5$, the activity exhibits nice oscillations at the input frequency. In order to have the same signal amplitude in the output, we should rather compare figure 5(c) with figure $5(d)$ which shows a network without connections $(J=0)$ but with a reduced $J_{\text {ext }}$.

Another way of looking at these results (figure 5) is in terms of the effects of inhibition on the dynamic range of the network activity. Figures $5(b)$ and $(c)$ show that, for the same $\mathrm{SNR}$, the dynamic range is improved when inhibition is present. This means that, due to 
a)

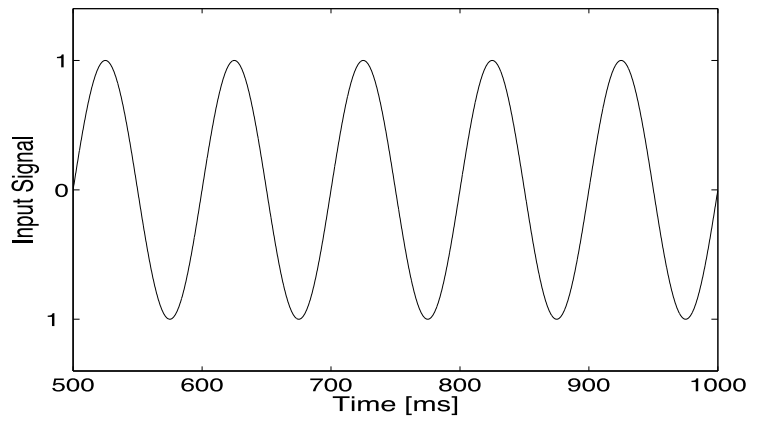

b)

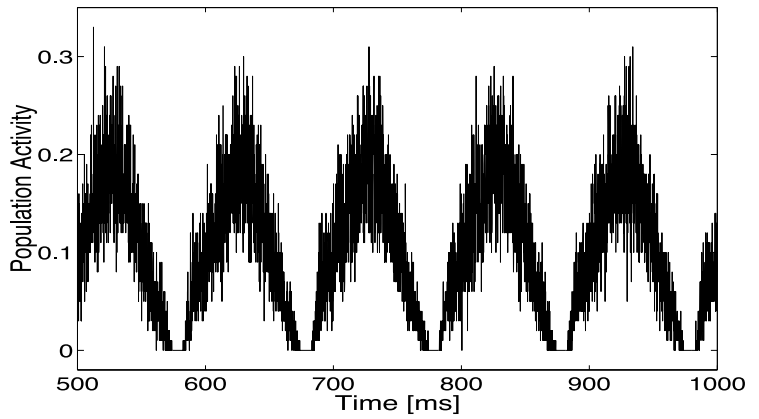

c)

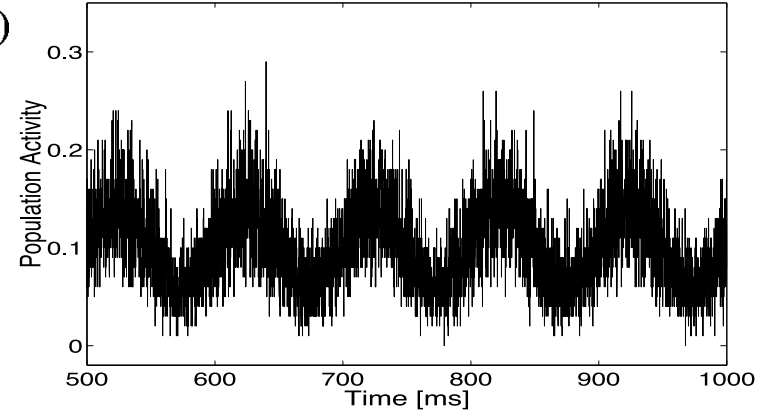

d)

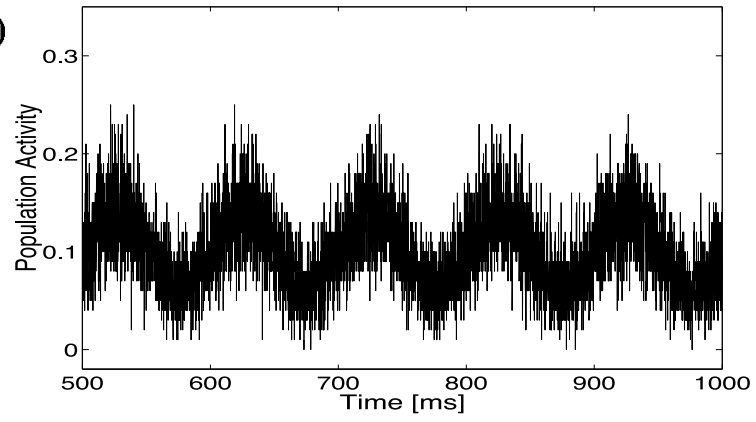

Figure 5. Neuronal population activity produced by a varying external signal. (a) Sinusoidal external input at $10 \mathrm{~Hz}$ frequency. (b) Population activity in the absence of coupling ( $J=0$ and $\left.J_{\text {ext }}=0.1\right)$. (c) Population activity for inhibitory couplings $(J=-5)$ with the same amplification (same $\left.J_{\text {ext }}\right)$ as in $(b)$. (d) Population activity in the absence of coupling with the same amplitude modulation as in $(c)\left(J_{\mathrm{ext}}=0.05\right)$. 
a)

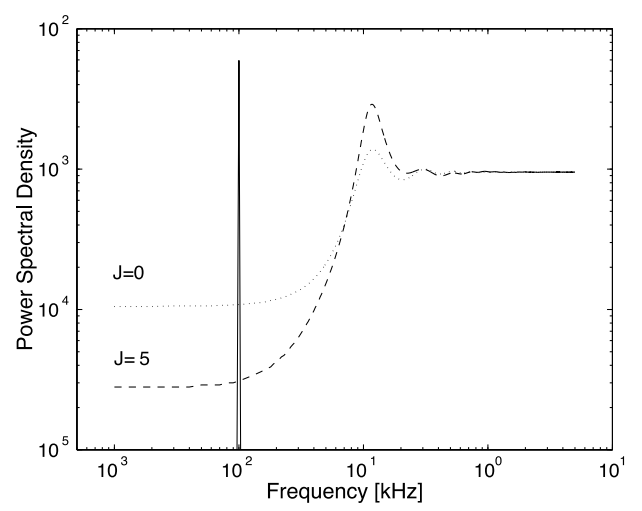

b)

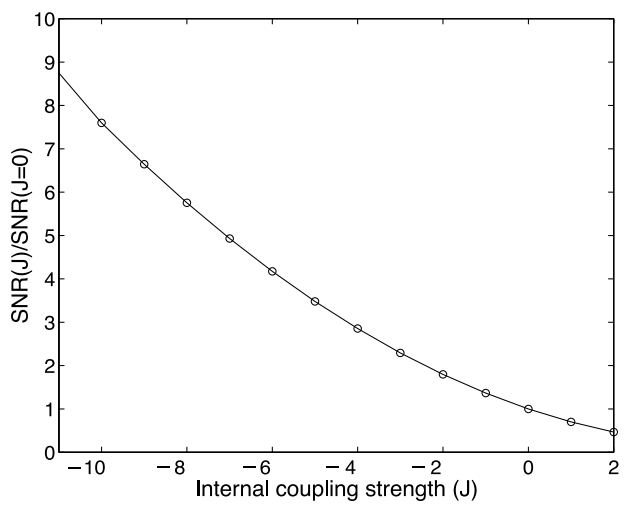

Figure 6. (a) Output signal power (solid line) in response to a $10 \mathrm{~Hz}$ input signal and noise power in the absence of couplings (dotted curve) and with inhibitory couplings (dashed curve). (b) Ratio between the SNR for a given internal coupling strength $(J)$ and the SNR without couplings $(J=0)$ plotted as a function of the internal coupling strength $(J)$. In the framework of the linear theory, the ratio does not depend on the input amplitude.

anticorrelations, signals with broad range of input amplitudes can be transmitted without large distortions.

\subsection{Information transmission}

If we assume that the system responds linearly to the input, the output signal power is given by equation (22) and does not depend on the noise level. Figure 6(a) plots the output signal spectrum and the noise spectrum for two different coupling strengths $(J=0$ and $J=-5)$. The noise spectrum has been calculated from equation (20). The output signal spectrum corresponds to the population activity spectrum of a network receiving a delta pulse in frequency at $10 \mathrm{~Hz}$ in the limit of $N \rightarrow \infty$ (equation (22)). The external coupling strength $\left(J_{\text {ext }}\right)$ has been adjusted to obtain the same output signal amplitude in both cases. Inhibitory couplings increase the SNR at the input frequency, because the noise level is reduced at this frequency (figure 6). With an internal coupling strength at $J=-5$, the SNR is approximatively 3.5 times higher than in the absence of coupling $(J=0)$.

From the SNR, we can estimate the amount of information transmitted at a given frequency by the system. In the linear regime, if the noise has a Gaussian distribution, the mutual information between the input signal and the output activity is always less than $[11,24,25]$ :

$$
I(\omega) \leqslant \frac{1}{2} \log _{2}[1+\operatorname{SNR}(\omega)] .
$$

If, in addition, the input signal is chosen from a Gaussian distribution, equation (25) becomes an equality. Thus, anticorrelations in the firings due to lateral inhibition increase the amount of information transmitted at low frequency.

\section{Discussion}

We have presented a theoretical analysis that describes the noise properties and the SNR in the frequency domain for a fully connected and homogeneous network. Neurons are described by the spike response model, which is a variant of the integrate-and-fire model. Noise is represented by an escape rate. Spectral noise shaping [4] due to coupling has previously been 
studied for fully connected homogeneous networks with absolute refractoriness [19] and also for networks of integrate-and-fire neurons with heterogeneous driving current [21].

The theory presented here also fits the noise spectrum of a partially connected network to a high degree of accuracy. We have searched for network parameters that modify the noise profile in the frequency domain. We have found that refractoriness and anticorrelations in the firings move noise from low to high frequencies. The same characteristics are also present in other noise models, like the noisy input model (see section 4.3). Moreover, similar results are found in inhomogeneous networks or networks with a noisy reset model [14] (data not shown). Thus, noise shaping seems to be a rather general property of neuronal networks.

Refractoriness reduces the noise at the low-frequency band by producing more regular spike trains. Indeed, if the mean firing rate is kept constant, refractoriness decreases the variance of the interspike interval. To understand why this produces a reduction of noise at low frequency, let us take the situation of a large refractory period. For large refractory periods, the network will tend to oscillate at the frequency given by the mean firing rate. The noise spectrum therefore exhibits peaks at this frequency and at its harmonics. Since the total noise power is constant, the effect of the peaks is to decrease the noise level at frequencies between the frequency peaks of oscillations (figure 2).

The effect of inhibition on the power spectrum can be understood with intuitive arguments. Neurons that fire will force other neurons in the network to remain silent. A short time later, many neurons are silent which decreases the overall inhibition. The probability of firing is therefore increased. Thus, the network activity changes at a high frequency between high and low firing probability. The low-frequency components of the noise are moved to higher frequencies. This means that anticorrelations act as a high-pass filter. On the other hand, if the inhibition is too strong, this may cause neurons to synchronize their activity. The network will tend to oscillate at the characteristic frequency of the system $[8,33]$.

The PSP time constants also affect the shape of the spectrum. Short time constants slightly increase the noise level at low frequency but also increase the bandwidth of the low-noise regime (data not shown). For high noise levels, the effect of the axonal delay on the noise spectrum is negligible. On the other hand, for low levels of noise the stability of the asynchronous state depends on the value of the delay $[14,15]$. Close to the instability, oscillations decay slowly which shows up in the spectrum by regular peaks (data not shown).

Some researchers [28,31] have found that spectra of the recorded activity of single neurons often present a $1 / f$ behaviour at low frequency, which contradicts our results. This behaviour, however, does not reflect the effect of refractoriness that produces a dip at low frequency. We speculate that the $1 / f$ dependence may be caused by correlated variability of the neuronal activity with external inputs from other cortical areas. Moreover, the noise shaping is a property of a population of neurons and not of a single neuron. In order to observe it experimentally, one should simultaneously measure the activity of many neurons, a technique that is not yet available.

Anticorrelations improve the SNR in a frequency range that can extend beyond the singleneuron frequency. This means that inputs that vary faster than the mean firing rate of a single neuron can still be transmitted with high SNR. Figure 7 gives a good illustration of this statement. A population of neurons spiking at rates between 3 and $13 \mathrm{~Hz}$ receives a $40 \mathrm{~Hz}$ external input signal (figure $7(a)$ ). The population activity generated by the signal looks rather noisy (figure $7(b)$ ). Now, let us take the point of view of a neuron receiving input from the first population. The membrane potential will show a low-pass filtered version of the activity. The filtered activity clearly shows the $40 \mathrm{~Hz}$ modulation (figure 7(c)). This modulation cannot be seen from the spike train of a single neuron in the population (figure $7(d)$ ).

In this paper, noise shaping is produced by fully inhibitory networks, which is, of course, 
(a)

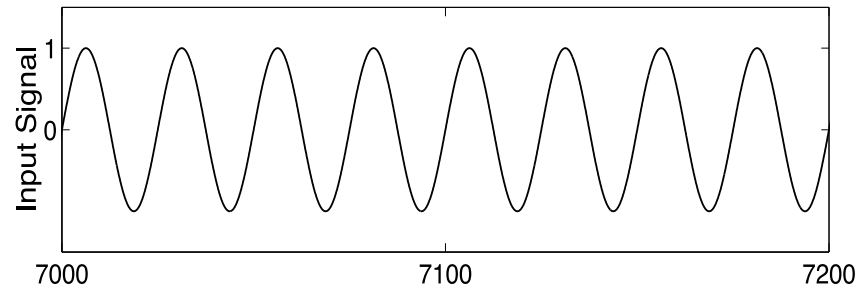

(b)

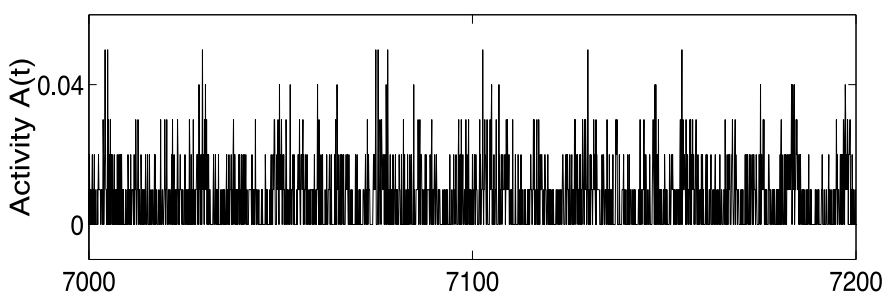

(c)

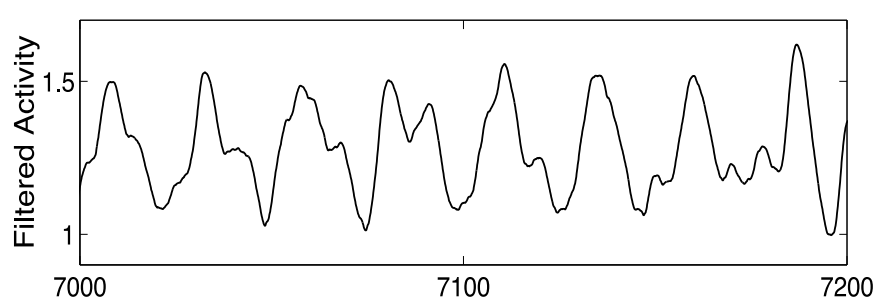

(d)

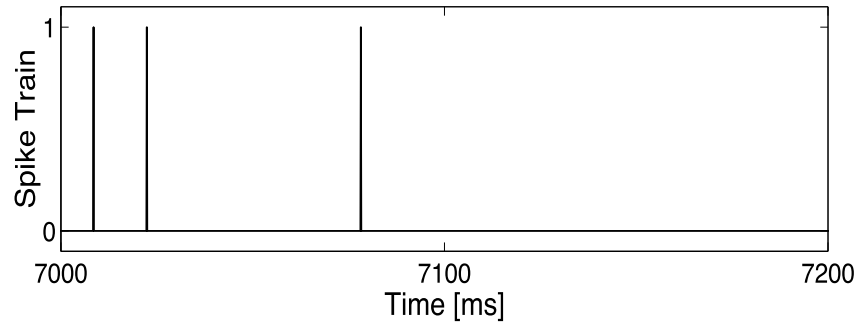

Figure 7. (a) A sinusoidal $40 \mathrm{~Hz}$ signal is applied to a network of 1000 neurons. (b) The population activity produced by the signal looks noisy. $(c)$ A neuron receiving input from the population will filter the activity due to its slow time constants. $(d)$ The spike train frequency of a single neuron in the population is much lower than the $40 \mathrm{~Hz}$ signal.

not realistic. We think that other coupling architectures that anticorrelate the neuronal firings may also generate noise shaping. Cortical neurons often present a connectivity pattern that consists of a local inhibition and a long-range excitation [3]. It has been shown [1,22, 34] that, for constant stimuli, correlations can increase the amount of information transmitted by a population of neurons coding for some feature variable. Here, we have considered timedependent stimuli but restricted the analysis to a single homogeneous pool of neurons. The extension of the present work to more biologically plausible network architectures is now under investigation. 


\section{Appendix}

In order to derive the noise spectrum, equation (20), we rewrite equation (14):

$$
0=\frac{\mathrm{d}}{\mathrm{d} t} \int_{-\infty}^{t} S_{h}(t \mid \hat{t}) A(\hat{t}) \mathrm{d} \hat{t}
$$

where we have used equation (11). The effect of the finite-size fluctuations is represented by a global multiplicative noise in an infinite system. Expression (16) is repeated here for convenience:

$$
\rho_{h}(t \mid \hat{t}) \longrightarrow \rho_{h}(t \mid \hat{t})[1+\sigma \xi(t)]
$$

where $\xi$ is a normally distributed random variable with zero mean and $\delta$-correlations. The fluctuating rate (27) causes fluctuations $\Delta A(t)$ in the activity which in turn cause fluctuations in the postsynaptic potential, $h(t)=h_{0}+\Delta h(t)$, where $h_{0}=J A_{0}+\mathrm{h}_{\mathrm{e}}$ and $\Delta h(t)=$ $J \int_{0}^{\infty} \epsilon(s) \Delta A(t-s) \mathrm{d} s+\Delta h_{\mathrm{ext}}(t)$. In order to calculate the spectrum of the fluctuations, we linearize equation (26) with respect to $\sigma$ and $\Delta h$. We find:

$$
\begin{aligned}
0=\frac{\mathrm{d}}{\mathrm{d} t} \int_{-\infty}^{t} S_{h=h_{0}, \sigma=0}(t \mid \hat{t}) \Delta A(\hat{t})+A_{0} \frac{\mathrm{d}}{\mathrm{d} t}\left\{\left.\int_{-\infty}^{t} \mathrm{~d} t_{1} \int_{-\infty}^{t} \mathrm{~d} \hat{t} \Delta h\left(t_{1}\right) \frac{\partial S_{h, \sigma=0}(t \mid \hat{t})}{\partial h\left(t_{1}\right)}\right|_{\Delta h=0}\right\} \\
+\left.A_{0} \frac{\mathrm{d}}{\mathrm{d} t} \int_{-\infty}^{t} \sigma \frac{\partial S_{h=h_{0}, \sigma}(t \mid \hat{t})}{\partial \sigma}\right|_{\sigma=0} \cdot
\end{aligned}
$$

With the relation (11) and $S_{h_{0}}(0)=1$ and the definition of the 'survivor' function $S_{h}$ (see section 2.2), we have:

$$
\begin{array}{rl}
\Delta A(t)=\int_{-\infty}^{t} & \mathrm{~d} \hat{t} P_{h_{0}}(t \mid \hat{t}) \Delta A(\hat{t}) \\
& +A_{0} \frac{\mathrm{d}}{\mathrm{d} t}\left[\int_{0}^{\infty} \mathrm{d} x \int_{x}^{\infty} \mathrm{d} s \Delta h(t-x)\left[\frac{\mathrm{d}}{\mathrm{d} h_{0}} \rho_{h_{0}}(s-x)\right] S_{h_{0}}(s)\right] \\
& +\sigma A_{0} \frac{\mathrm{d}}{\mathrm{d} t}\left[\int_{0}^{\infty} \mathrm{d} x \int_{x}^{\infty} \mathrm{d} s \xi(t-x) \rho_{h_{0}}(s-x) S_{h_{0}}(s)\right] .
\end{array}
$$

We now use the filters $\mathcal{F}$ and $\mathcal{L}$ defined in (18) and (19). This gives equation (17). The result in the Fourier domain is

$$
\Delta A(\omega)=\tilde{P}_{h_{0}}(\omega) \Delta \tilde{A}(\omega)+\mathrm{i} \omega A_{0} \tilde{\mathcal{L}}(\omega) \Delta \tilde{h}(\omega)+\mathrm{i} \omega \sigma A_{0} \tilde{\mathcal{F}}(\omega) \tilde{\xi}(\omega) .
$$

In the absence of a varying external input $\left(\Delta h_{\text {ext }}(t)=0\right)$, we have $\Delta \tilde{h}(\omega)=$ $J \tilde{\varepsilon}(\omega) \Delta \tilde{A}(\omega)$. Equation (30) reduces to

$\Delta A(\omega)=\tilde{P}_{h_{0}}(\omega) \Delta \tilde{A}(\omega)+\mathrm{i} \omega A_{0} \tilde{\mathcal{L}}(\omega) J \tilde{\mathcal{E}}(\omega) \Delta \tilde{A}(\omega)+\mathrm{i} \omega \sigma A_{0} \tilde{\mathcal{F}}(\omega) \tilde{\xi}(\omega)$

The Fourier transform of equation (11) gives: $P_{h_{0}}(\omega)=-\mathrm{i} \omega S_{h_{0}}(\omega)+1$. Using this relation, we find the analytical expression of the noise spectrum given by equation (20).

\section{References}

[1] Abbott L F and Dayan P 1999 The effect of correlated variability on the accuracy of a population code Neural Comput. 1 91-101

[2] Abbott L F and Van Vreeswijk C 1993 Asynchronous states in networks of pulse-coupled oscillators Phys. Rev. E 481483

[3] Abeles M 1991 Corticonics (Cambridge: Cambridge University Press)

[4] Adams R W 1997 Spectral noise-shaping in integrate-and-fire neural networks Proc. Int. Conf. on Neural Networks (ICNN '97) (Houston, TX) (Los Alamitos, CA: IEEE Computer Society Press) p 953 
[5] Bair W, Koch C, Newsome W and Britten K 1994 Power spectrum analysis of MT neurons in the behaving monkey J. Neurosci. 14 2870-92

[6] Brunel N and Hakim V 1999 Fast global oscillations in networks of integrate-and-fire neurons with low firing rates Neural Comput. 11 1621-71

[7] Bryant H L and Segundo J P 1976 Spike inititation by transmembrane current: a white noise analysis J. Physiol. $260279-314$

[8] Chow C C 1997 Phase-locking in weakly heterogeneous neuronal networks Physica D 118 343-70

[9] Cox D R 1962 Renewal Theory (London: Mathuen)

[10] Daley D and Vere-Jones D 1988 An Introduction to the Theory of Point Processes (New York: Springer)

[11] Deco G and Obradovic D 1996 An Information-Theoretic Approach to Neural Computing (New York: Springer)

[12] Edwards B E and Wakefield G H 1993 The spectral shaping of neural discharges by refractory effects J. Acoust. Soc. Am. 93 3553-64

[13] Gerstner W 1995 Time structure of the activity in neural network models Phys. Rev. E 51 738-58

[14] Gerstner W 1999 Population dynamics for spiking neurons: fast transients, asynchronous states and locking Neural Comput. 11 1875-921

[15] Gerstner W, Van Hemmen J 1 and Cowan J D 1996 What matters in neuronal locking Neural Comput. 8 1689-712

[16] Gerstner W and Van Hemmen J L 1992 Associative memory in a network of spiking neurons Network 3 139-64

[17] Franklin J and Bair W 1995 The effect of a refractory period on the power spectrum of neuronal discharge SIAM J. Appl. Math. 55 1074-93

[18] Knight B W 1972 Dynamics of encoding in a population of neurons J. Gen. Physiol. 59 734-66

[19] Spiridon M, Chow C C and Gerstner W 1998 Frequency spectrum of coupled stochastic neurons with refractoriness Proc. 8th Int. Conf. on Artificial Neural Networks (ICANN '98) vol 1, ed M Boden, L Niklasson and T Ziemke (Berlin: Springer) pp 337-42

[20] Mainen Z F and Sejnowski T J 1995 Reliability of spike timing in neocortical neurons Science 268 $1503-6$

[21] Mar D J, Chow C C, Gerstner W, Adams R W and Collins J J 1999 Noise-shaping in populations of coupled model neurons Proc. Natl Acad. Sci. USA 96 to appear

[22] Panzeri S, Schultz S R and Treves A 1999 Correlations and encoding of information in the nervous system Proc. R. Soc. B 266 1001-12

[23] Plesser H E and Gerstner W 1999 Stochastic resonance in integrate-and-fire neurons: comparison of noisemodels Neural Comput. to appear

[24] Rieke F, Warland D, De Ruyter van Steveninck R and Bialek W 1997 Spikes-Exploring the Neural Code (Cambridge, MA: MIT)

[25] Shannon C E 1948 A mathematical theory of communication Bell Syst. Tech. J. 27 623-56

[26] Softky W R and Koch C 1993 The highly irregular firing of cortical cells is inconstitent with temporal intergration of random EPSPs J. Neurosci. $13334-50$

[27] Thorpe S, Fize D and Marlot C 1996 Speed of processing in the human visual system J. Comput. Neurosci. 381 $520-2$

[28] Tovee M J, Rolls E T, Treves A and Belles R P 1993 Information encoding and the responses of single neurons in the primate visual cortex J. Neurophysiol. $70640-54$

[29] Treves A 1993 Mean-field analysis of neuronal spike dynamics Network 4 259-84

[30] Tsodyks M V and Sejnowski 1995 Rapid state switching in balanced cortical network models Comput. Neural Syst. 6 111-24

[31] Usher M, Stemmler M and Olami Z 1995 Dynamic pattern formation leads to $1 / f$ noise in neural populations Phys. Rev. Lett. 74 326-9

[32] Van Vreeswijk C and Sompolinsky H 1996 Chaos in neuronal networks with balanced excitatory and inhibitory activity Science 274 1724-6

[33] Van Vreeswijk C, Abbott L F and Bard Ermentrout G 1994 When inhibition not excitation synchronizes neural firing J. Comput. Neurosci. $1313-21$

[34] Yoon H and Sompolinsky H 1999 Population Coding with Correlated Noise (Advances in Neural Information Processing Systems vol 11) ed M I Jordan, M J Kearns and S A Solla (Cambridge, MA: MIT) 